\title{
Existence, Uniqueness, and Almost Sure Exponential Stability of Solutions to Nonlinear Stochastic System with Markovian Switching and Lévy Noises
}

\author{
Chao Wei \\ School of Mathematics and Statistic, Anyang Normal University, Anyang 455000, China \\ Correspondence should be addressed to Chao Wei; chaowei0806@aliyun.com
}

Received 30 March 2020; Accepted 26 May 2020; Published 20 June 2020

Academic Editor: Sigurdur F. Hafstein

Copyright (c) 2020 Chao Wei. This is an open access article distributed under the Creative Commons Attribution License, which permits unrestricted use, distribution, and reproduction in any medium, provided the original work is properly cited.

\begin{abstract}
This paper is concerned with existence, uniqueness, and almost sure exponential stability of solutions to nonlinear stochastic system with Markovian switching and Lévy noises. Firstly, the existence and uniqueness of solutions to the system is studied. Then, the almost sure exponential stability of the system is derived. Finally, an example is presented to illustrate the results.
\end{abstract}

\section{Introduction}

In practical engineering applications, some linear systems may be affected by external factors or internal causes to appear nonlinear phenomena, such as deformation of the spring due to excessive external force, sensor measurement due to environmental influences, or their own causes leading to the input and output show a nonlinear relationship. This makes nonlinearity an important factor that cannot be ignored. On the contrary, almost all dynamic systems are nonlinear. If the input and output of the system are not proportional, the system can be regarded as a nonlinear system. Moreover, most systems are affected by some kind of noise, and such noise usually shows a certain random characteristic. Therefore, it is reasonable and necessary to model the actual system with a nonlinear stochastic system. With the development of science and technology, nonlinear stochastic systems have attracted the attention of many scholars $([1,2])$. However, in engineering practice, most random disturbances exhibit non-Gauss characteristics. In recent years, with the development of the Lévy process theory, Lévy noise, as an important type of non-Gauss noise, has been widely used in engineering systems, economic systems, and management systems and has attracted great attention from scholars. Bertoin [3] introduced the basic theory of the Lévy process. Applebaum [4] introduced stochastic integral and stochastic differential equation driven by Lévy process. Applebaum [5] proposed that Lévy noise can be decomposed into a continuous part and a jump part which, respectively, corresponds to the diffusion and jump term in systems by Lévy-Itô decomposition. Wong and Guan [6] used fast Fourier transform to solve option pricing in Lévy process. Liao et al. [7] studied pricing power options with generalized jump diffusion.

During actual project operation, the system may appear between systems described by homogeneous models with different coefficients due to factors such as component failures and repairs, changes in the connection methods of subsystems, and changes in operating points of nonlinear systems after linearization [8]. Obviously, the deterministic model cannot accurately represent this switching relationship between different subsystems nor can it achieve the effect expected in engineering. By studying the nature of the Markov process, the scholars found that it can well reflect this switching relationship between subsystems, and it has been introduced to simulate such systems. In general, the state of a continuous Markov transition system consists of two parts. One part is the modal state of the system, and the other part is the system state. Therefore, the Markov transition system is sometimes called the hybrid system. Over the past few decades, many authors studied the systems with Markovian switching. For example, Xi [9] 
studied the stability of system with Markovian switching and jump diffusions. Huang and Mao [10] applied Holder's inequality, Chebyshev's inequality, and Borel-Cantelli's lemma to analyze the almost sure stability of nonlinear time delayed stochastic systems with Markovian switching and jump diffusion. Zhou et al. [11] studied slide mode and delay-dependent adaptive exponential synchronization in $p$ th moment for stochastic delayed neural networks with Markovian switching. Wu et al. [12] discussed the stabilization of stochastic coupled systems with Markovian switching via feedback control based on discrete-time observations.

For any practical system, it will always be disturbed by various random factors during its normal operation. In this case, whether the system can maintain the predetermined working state safely is a basic and important research content. In the past few decades, the problem of stability of systems has been investigated by many authors [13-16]. For example, Liu et al. [17] constructed an equivalent set of delay differential algebraic equations to study the stability of systems modeled as neutral delay differential equations. Wu et al. [18] analyzed the almost sure globally asymptotical stability and almost sure exponential stability for switched systems with semi-Markovian switching, Markovian switching, and renewal process switching signals, respectively. Li et al. [19] used piecewise constant Lyapunov matrices and the generalized Kalman-Yakubovich-Popov lemma to discuss strong delay-independent stability of linear time-invariant systems. Song and $\mathrm{Xu}[20]$ investigated the stability of the metastable low and high concentration states under nonGaussian noise based on the stochastic basin of attraction. However, there are few literature studies about the existence, uniqueness, and almost sure exponential stability of solutions to nonlinear stochastic system with Markovian switching and Lévy noises. This paper uses probability analysis methods to study the existence and uniqueness of global solution to nonlinear stochastic system with Markovian switching and Lévy noises under the condition of local Lipschitz condition and local linear growth condition. Then, the almost sure exponential stability of the solution is proved by the generalized Itô formula, the law of large numbers for local martingale, and the ergodic of Markovian chain.

This paper is organized as follows. In Section 2, we introduce the nonlinear stochastic system with Markovian switching and Lévy noises. Some important assumptions, definitions, and lemmas are given as well. In Section 3, the existence and the almost sure exponential stability of the global unique solution to the system are studied. In Section 4 , a numerical example is provided. The conclusion is given in Section 5.

\section{Problem Formulation and Preliminaries}

Let $(\Omega, \mathscr{F}, \mathbb{P})$ be a complete probability space equipped with a right continuous and increasing family of $\sigma$-algebras $\left(\left\{\mathscr{F}_{t}\right\}_{t \geq 0}\right)$. Denoted by $\mathscr{C}^{1,2}\left(\mathbb{R}^{n} \times \mathbb{R}_{+} \times \mathbb{S} ; \mathbb{R}_{+}\right)$, the family of positive real-valued functions $V(x, t, i)$ defined on
$\mathbb{R}^{n} \times \mathbb{R}_{+} \times \mathbb{S}$ are continuously twice differentiable in $x \in \mathbb{R}^{n}$ and once differentiable in $t \in \mathbb{R}_{+}$. Let $r(t), t \geq 0$ be a rightcontinuous Markov chain on the probability space taking values in a finite state space $\mathbb{S}=\{1,2, \ldots, N\}$ with generator $\Gamma=\left(\gamma_{i j}\right)_{N \times N}$ given by

$$
\mathbb{P}\{r(t+\Delta)=j \mid r(t)=i\}= \begin{cases}\gamma_{i j} \Delta+o(\Delta), & i \neq j, \\ 1+\gamma_{i i} \Delta+o(\Delta), & i=j,\end{cases}
$$

where $\Delta>0$ and $\gamma_{i j} \geq 0$ is the transition rate from $i$ to $j$ if $i \neq j$ while $\gamma_{i i}=-\sum_{i \neq j} \gamma_{i j}$.

We consider the following nonlinear stochastic system:

$$
\begin{aligned}
\mathrm{d} x(t)= & f(x(t), t, r(t)) \mathrm{d} t+g(x(t), t, r(t)) \mathrm{d} B(t) \\
& +\int_{Y} G(x(t-), t, r(t-), y) N(\mathrm{~d} t, \mathrm{~d} y),
\end{aligned}
$$

where $x(0)=x_{0} \in \mathscr{F}_{0}, r(0)=r_{0} \in \mathbb{S}, x(t-)=\lim _{s \downarrow t} x(s)$, $B(t)$ is an $m$-dimensional standard Brownian motion, $N(t, y)$ is a Poisson random measure on $[0,+\infty) \times \mathbb{R}^{n}$ with compensator $\quad \tilde{N}(t, y) \quad$ which satisfies $\tilde{N}(t, y)=N(\mathrm{~d} t, \mathrm{~d} y)-v(\mathrm{~d} y) \mathrm{d} t, v(\mathrm{~d} y)$ is a Lévy measure, $f: \mathbb{R}^{n} \times \mathbb{R}_{+} \times \mathbb{S} \longrightarrow \mathbb{R}^{n}, \quad g: \mathbb{R}^{n} \times \mathbb{R}_{+} \times \mathbb{S} \longrightarrow \mathbb{R}^{n \times m}, \quad$ and $G: \mathbb{R}^{n} \times \mathbb{R}_{+} \times \mathbb{S} \times \mathbb{R}^{n} \longrightarrow \mathbb{R}^{n}$. We assume that $B(t)$, $N(t, y)$, and $r(t)$ in system (1) are independent, and Markov chain is ergodic. Then, Markov chain has a unique stable distribution $\pi=\left(\pi_{1}, \pi_{2}, \ldots, \pi_{N}\right) \in \mathbb{R}^{1 \times N} . \pi$ can be solved by the following equation:

$$
\pi \Gamma=0 \quad \text { s.t. } \sum_{j=1}^{N} \pi_{j}=1, \quad \forall j \in \mathbb{S} .
$$

We put the following assumptions, definitions, and lemmas.

Assumption 1. For each $K>0$, there exists $L_{K}>0$ such that, for any $t \geq 0,\left|x_{1}\right| \vee\left|x_{2}\right| \leq K$ and $i \in S, \mid f\left(x_{1}, t, i\right)-f\left(x_{2}\right.$, $t, i)\left.\right|^{2}+\left|g\left(x_{1}, t, i\right)-g\left(x_{2}, t, i\right)\right|^{2}+\int_{Y} \mid G\left(x_{1}, t, i, y\right)-G\left(x_{2}, t\right.$, $i, y)\left.\right|^{2} \nu(\mathrm{d} y) \leq L_{k}$.

Assumption 2. There exists $V(x, t, i) \in \mathscr{C}^{1,2}\left(\mathbb{R}^{n} \times \mathbb{R}_{+} \times\right.$ $\left.\mathbb{S} ; \mathbb{R}_{+}\right) \quad$ and $m(s) \in L^{1}\left(\mathbb{R}_{+} ; \mathbb{R}_{+}\right)$such that $\lim _{|x| \longrightarrow \infty} \inf _{t \geq 0, i \in S} V(x, t, i)=\infty$ and $\mathscr{L} V(x, t, i) \leq m(s)$.

Assumption 3. $f(0, t, i) \equiv 0, g(0, t, i) \equiv 0, G(0, t, i, y) \equiv 0$, $\forall i \in \mathbb{S}$, and $\forall y \in Y$.

Definition 1. The solution of system (1) is said to be almost sure exponential stability if there exists $\lambda>0$ satisfying

$$
\lim _{t \longrightarrow \infty} \sup \frac{1}{t} \log \left(\left|x\left(t ; x_{0}, r_{0}\right)\right|\right)<0,
$$

for any $x_{0} \in \mathscr{F}_{0}$ and $r_{0} \in \mathbb{S}$.

Given $V \in \mathscr{C}^{1,2}\left(\mathbb{R}^{n} \times \mathbb{R}_{+} \times \mathbb{S} ; \mathbb{R}_{+}\right)$, we define the operator $\mathscr{L} V$ by 


$$
\begin{aligned}
\mathscr{L} V(x, t, i)= & V_{t}(x, t, i)+V_{x}(x, t, i) f(x, t, i) \\
& +\frac{1}{2} \operatorname{trace}\left[g^{T}(x, t, i) V_{x x}(x, t, i) g(x, t, i)\right] \\
& +\int_{Y} \sum_{k=1}^{l}\left[V\left(x+G^{k}\left(x, t, i, y_{k}\right), t, i\right)\right. \\
& -V(x, t, i) v_{k}\left(\mathrm{~d} y_{k}\right)+\sum_{j=1}^{N} \gamma_{i j} V(x, t, j) .
\end{aligned}
$$

Lemma 1 (see [21]). Let $H=\{H(t)\}_{t \geq 0}$ be a real-valued local martingale and $H(0)=0$, then

$$
\begin{gathered}
\lim _{t \longrightarrow \infty} \int_{0}^{t} \frac{\mathrm{d}\langle H, H\rangle s}{(1+s)^{2}}<\infty, \quad \text { a.s. } \\
\Rightarrow \lim _{t \longrightarrow \infty} \frac{H(t)}{t}=0, \quad \text { a.s. }
\end{gathered}
$$

Remark 1. According to Assumption 1, it is easy to check that system (1) satisfies local linear growth condition. Since, the local solution of system (1) exists and is unique. In the next section, we prove that the global unique solution exists.

\section{Existence, Uniqueness, and Almost Sure Exponential Stability of Solutions to Nonlinear Stochastic System with Markovian Switching and Lévy Noises}

In the following theorem, the existence and uniqueness of solutions to nonlinear stochastic system with Markovian switching and Lévy noises are derived.

Theorem 1. Suppose that Assumptions 1 and 2 hold. Then, the system has a global unique solution $\{x(t), t \geq 0\}$.

Proof. For the given initial values $x_{0}$ and $r_{0}$, we assume that $\left|x_{0}\right| \leq \eta$. For $k \geq \eta, k \in \mathbb{N}$, let

$$
\begin{aligned}
& f^{(k)}(x, t, i)=f\left(\frac{|x| \wedge k}{|x|} x, t, i\right), \\
& g^{(k)}(x, t, i)=g\left(\frac{|x| \wedge k}{|x|} x, t, i\right),
\end{aligned}
$$

where $((|x| \wedge k /|x|) x)=0$ when $x=0$.

It is easy to check that $f^{(k)}$ and $g^{(k)}$ satisfy the local Lipschitz condition and linear growth condition. Then, we obtain that

$$
\begin{aligned}
\mathrm{d} x_{k}(t)= & f^{(k)}\left(x_{k}(t), t, r(t)\right) \mathrm{d} t+g^{(k)}\left(x_{k}(t), t, r(t)\right) \mathrm{d} B(t) \\
& +\int_{Y} G x_{k}(t-), t, r(t-) N(\mathrm{~d} t, d y)
\end{aligned}
$$

has the global unique solution.

For $k \in \mathbb{N}$, we define the stopping time:

$$
\alpha_{k}=\inf \left\{t \geq 0:\left|x_{k}(t)\right| \geq k\right\},
$$

where $\inf \phi=\infty$.

It can be checked that when $0 \leq t \leq \alpha_{k}$ and $x_{k}(t)=x_{k+1}$, namely, $\left\{\alpha_{k}\right\}$ is increasing. Therefore, there exists a stopping time $\alpha$ such that

$$
\alpha=\lim _{n \longrightarrow \infty} \alpha_{k}
$$

Define

$$
x(t)=\lim _{k \longrightarrow \infty} x_{k}(t), \quad 0 \leq t<\alpha .
$$

Obviously, when $0 \leq t<\alpha, x(t)$ is the unique solution of system (1).

We now show that $x(t)$.

For $t \geq 0$, according to Itô formula, we have

$$
\begin{aligned}
& \mathbb{E} V\left(x_{k}\left(t \wedge \alpha_{k}\right), t \wedge \alpha_{k}, r\left(t \wedge \alpha_{k}\right)\right) \\
& \quad=\mathbb{E} V\left(x_{k}(0),(0), r(0)\right)+\mathbb{E} \int_{0}^{t \wedge \alpha_{k}} \mathscr{L}^{(k)} V\left(x_{k}(s), s, r(s)\right) \mathrm{d} s,
\end{aligned}
$$

where $\mathscr{L}^{(k)} V\left(x_{k}(s), s, r(s)\right)=\mathscr{L} V\left(x_{k}(s), s, r(s)\right) \quad$ when $0 \leq s \leq t \wedge \alpha_{k}$.

Then, from Assumption 1, we obtain

$$
\begin{aligned}
& \mathbb{E} V\left(x_{k}\left(t \wedge \alpha_{k}\right), t \wedge \alpha_{k}, r\left(t \wedge \alpha_{k}\right)\right) \\
& \leq \mathbb{E} V\left(x_{0}, 0, r_{0}\right)+\int_{0}^{t} m(s) \mathrm{d} s \\
& <\infty .
\end{aligned}
$$

Moreover, since

$$
\begin{array}{r}
P\left\{\alpha_{k} \leq t\right\} \inf _{|x| \geq n, t \geq 0, i \in S} V(x, t, i) \\
\leq \int_{\alpha_{k} \leq t} V\left(x_{k}\left(t \wedge \alpha_{k}\right), t \wedge \alpha_{k}, r\left(t \wedge \alpha_{k}\right)\right) \mathrm{d} P \\
\leq \mathbb{E} V\left(x_{k}\left(t \wedge \alpha_{k}\right), t \wedge \alpha_{k}, r\left(t \wedge \alpha_{k}\right)\right),
\end{array}
$$

we have

$$
P\left\{\alpha_{k} \leq t\right\} \leq \frac{\mathbb{E} V\left(x_{0}, 0, r_{0}\right)+\int_{0}^{t} m(s) \mathrm{d} s}{\inf _{|x| \geq n, t \geq 0, i \in S} V(x, t, i)} .
$$

When $t \longrightarrow \infty$, it follows that

$$
P\{\alpha \leq t\}=0 .
$$

Therefore,

$$
P\{\alpha=\infty\}=1 .
$$

The proof is complete.

In the following theorem, the almost sure exponential stability for the nonlinear stochastic system with Markovian switching and Lévy noises is derived. The conditions of stability only depend on the state of Markovian chain and some constants. 
Theorem 2. Suppose that Assumptions 1 and 3 hold. For any $x(t) \in \mathbb{R}^{n}$, if there exists a symmetric positive definite matrix $F$ and some constants $\gamma_{i} \in \mathbb{R}, \delta_{i}, \xi_{i}, \sigma_{i} \geq 0,(i \in \mathbb{S})$ satisfy $2 x^{T} F f(x, t, i)+\operatorname{tr}\left(g^{T}(x, t, i) F g(x, t, i)\right) \leq \gamma_{i} x^{T} F x$,

$$
\begin{gathered}
\left|x^{T} F g(x, t, i)\right|^{2} \geq \delta_{i}\left(x^{T} F x\right)^{2}, \\
\xi_{i}|x| \leq|G(x, t, i, y)+x| \leq \sigma_{i}|x|
\end{gathered}
$$

$$
\begin{aligned}
& \log \left(x^{T}(t) F x(t)\right) \\
& =\log \left(x_{0}^{T} F x_{0}\right)+\int_{0}^{t}\left(\frac{2 x^{T}(s) F f(x(s), s, r(s))+\operatorname{tr}\left(g^{T}(x(s), s, r(s)) F g(x(s), s, r(s))\right)}{x^{T}(s) F x(s)}\right. \\
& \left.-\frac{2\left|x^{T}(s) F g(x(s), s, r(s))\right|^{2}}{\left(x^{T}(s) F x(s)\right)^{2}}+\sum_{j=1}^{N} \gamma_{i j} \log \left(x^{T}(t) F x(t)\right)\right) \mathrm{d} s \\
& +\int_{0}^{t} \frac{2 x^{T}(s) F g(x(s), s, r(s))}{x^{T}(s) F x(s)} \mathrm{d} B(s) \\
& +\int_{0}^{t} \int_{Y}\left(\operatorname { l o g } \left((x(s)+G H(x(s-), s, r(s-), y))^{T} F(x(s)+G(x(s-), s, r(s-), y))\right.\right. \\
& \left.-\log \left(x^{T}(s) F x(s)\right)\right) N(\mathrm{~d} s, \mathrm{~d} y) \\
& =\log \left(x_{0}^{T} F x_{0}\right)+\int_{0}^{t}\left(\frac{2 x^{T}(s) F f(x(s), s, r(s))+\operatorname{tr}\left(g^{T}(x(s), s, r(s) F g(x(s), s, r(s)))\right)}{x^{T}(s) F x(s)}-\frac{2 \mid x^{T}(s) F g(s)^{2}}{\left(x^{T}(s) F x(s)\right)^{2}}\right) \mathrm{d} s \\
& +\int_{0}^{t} \frac{2 x^{T}(s) F g(x(s), s, r(s))}{x^{T}(s) F x(s)} \mathrm{d} B(s) \\
& +\int_{0}^{t} \int_{Y} \frac{(\log x(s)+G(x(s-), s, r(s-), y))^{T} F(x(s)+G(x(s-), s, r(s-), y))}{x^{T}(s) F x(s)} \tilde{N}(\mathrm{~d} s, \mathrm{~d} y) \\
& +\int_{0}^{t} \int_{Y} \frac{(\log x(s)+G(x(s-), s, r(s-), y))^{T} F(x(s)+G(x(s-), s, r(s-), y))}{x^{T}(s) F x(s)} v(\mathrm{~d} y) \mathrm{d} s .
\end{aligned}
$$

Let

$$
\begin{aligned}
& H_{1}=\int_{0}^{t} \frac{2 x^{T}(s) F g(x(s), s, r(s))}{x^{T}(s) F x(s)} \mathrm{d} B(s), \\
& H_{2}=\int_{0}^{t} \int_{Y} \frac{\log \left(x(s)+G\left(x\left(s^{-}\right), s, r(s-), y\right)\right)^{T} F(x(s)+G(x(s-), s, r(s-), y))}{x^{T}(s) F x(s)} \tilde{N}(\mathrm{~d} s, \mathrm{~d} y) .
\end{aligned}
$$


It is obvious that $H_{1}$ and $H_{2}$ are local martingale.

Then, the quadratic variation of $H_{1}$ satisfies

$$
\begin{aligned}
& \int_{0}^{t} \frac{\mathrm{d}\left\langle H_{1}, H_{1}\right\rangle s}{(1+s)^{2}} \\
& \quad=\int_{0}^{t} \frac{4\left|x^{T}(s) F g(x(s), s, r(s))\right|^{2}}{\left(x^{T}(s) F x(s)\right)^{2}(1+s)^{2}} \mathrm{~d} s \\
& \quad \leq \int_{0}^{t} \frac{4 L_{k}|x(s)|^{4}\|F\|^{2}}{\lambda_{\min }^{2}(F)|x(s)|^{4}(1+s)^{2}} \mathrm{~d} s \\
& \quad \leq \frac{4 L_{k}\|F\|^{2}}{\lambda_{\min }^{2}(F)} \int_{0}^{\infty} \frac{1}{(1+s)^{2}} \mathrm{~d} s<\infty .
\end{aligned}
$$

From equation (12), we obtain

$$
\begin{aligned}
& \log \frac{\lambda_{\min }(F) \xi_{r(s-)}^{2}}{\lambda_{\max }(F)} \\
& \leq \log \frac{\lambda_{\min }(F) \mid x(s)+G(x(s-), s, r(s-), y) \|^{2}}{\lambda_{\max }(F)|x(s)|^{2}} \\
& \leq \log \frac{(x(s)+G(x(s-), s, r(s-), y))^{T} F(x(s)+G(x(s-), s, r(s-), y))}{x^{T}(s) F x(s)} \\
& \leq \log \frac{\lambda_{\max }(F)|x(s)+G(x(s-), s, r(s-), y)|^{2}}{\lambda_{\min }(F)|x(s)|^{2}} \\
& \leq \log \frac{\lambda_{\max }(F) \sigma_{r(s-)}^{2}}{\lambda_{\min }(F)} .
\end{aligned}
$$

Hence,

$$
\left|\log \frac{\left(x(s)+G\left(x\left(s^{-}\right), s, r\left(s^{-}\right), y\right)\right)^{T} F\left(x(s)+G\left(x\left(s^{-}\right), s, r(s-), y\right)\right)}{x^{T}(s) F x(s)}\right|^{2} \leq H,
$$

where $\quad H=\left|\log \left(\lambda_{\min }(F) \xi_{r(s-)}^{2} / \lambda_{\max }(F)\right)\right| \mathrm{V} \mid \log \left(\lambda_{\max }(F) \quad\right.$ Thus, $\left.\sigma_{r(s-)}^{2} / \lambda_{\min }(F)\right) \mid$.

$$
\begin{aligned}
& \int_{0}^{t} \frac{\mathrm{d}\left\langle H_{2}, H_{2}\right\rangle s}{(1+s)^{2}} \\
& \quad=\int_{0}^{t} \frac{\left|\log \left((x(s)+G(x(s-), s, r(s-), y))^{T} F(x(s)+G(x(s-), s, r(s-), y)) / x^{T}(s) F x(s)\right)\right|^{2}}{(1+s)^{2}} \mathrm{~d} s \\
& \quad \leq L \int_{0}^{\infty} \frac{1}{(1+s)^{2}} \mathrm{~d} s<\infty .
\end{aligned}
$$


Therefore, according to Lemma 1, it follows that

$$
\begin{aligned}
& \lim _{t \longrightarrow \infty} \frac{H_{1}(t)}{t}=0, \\
& \lim _{t \rightarrow \infty} \frac{H_{2}(t)}{t}=0 .
\end{aligned}
$$

From equation (12), we have

$$
\begin{aligned}
& \int_{0}^{t} \int_{Y} \log \frac{(x(s)+G(x(s-), s, r(s-), y))^{T} F(x(s)+G(x(s-), s, r(s-), y))}{x^{T}(s) F x(s)} v(\mathrm{~d} y) \mathrm{d} s \\
& \quad \leq \int_{0}^{t} \int_{Y} \log \frac{\lambda_{\max }(F)|x(s)+G(x(s-), s, r(s-), y)|^{2}}{\lambda_{\min }(F)|x(s)|^{2}} \nu(\mathrm{d} y) \mathrm{d} s \\
& \quad \leq \int_{0}^{t} \log \frac{\lambda_{\max }(F) \sigma_{r(s-)}^{2}}{\lambda_{\text {min }}(F)} \mathrm{d} s .
\end{aligned}
$$
that

Together with equations (10) and (11), it can be checked

$$
\begin{aligned}
& \log (\left.x^{T}(t) F x(t)\right) \\
& \leq \log \left(x^{T}(0) F x(0)\right)+H_{1}(t)+H_{2}(t) \\
& \quad+\int_{0}^{t}\left(\gamma_{r(s)}-2 \delta_{r(s)}+\log \frac{\lambda_{\text {max }}(F) \sigma_{r(s-)}^{2}}{\lambda_{\text {min }}(F)}\right) \mathrm{d} s .
\end{aligned}
$$

According to the ergodic of the Markovian chain, we have

$$
\begin{aligned}
\lim _{t \rightarrow \infty} & \frac{1}{t} \int_{0}^{t}\left(\gamma_{r(s)}-2 \delta_{r(s)}+\log \frac{\lambda_{\max }(F) \sigma_{r(s-)}^{2}}{\lambda_{\min }(F)}\right) \mathrm{d} s \\
& =\sum_{i=1}^{N} \pi_{i}\left(\gamma_{i}-2 \delta_{i}+\log \frac{\lambda_{\max }(F) \sigma_{i}^{2}}{\lambda_{\min }(F)}\right) .
\end{aligned}
$$

From equations (15)-(18), we obtain

$$
\begin{aligned}
\lim _{t \rightarrow \infty} \sup \frac{1}{t} \log (|x(t)|) \\
\quad=\frac{1}{2} \lim _{t \rightarrow \infty} \sup \frac{1}{t} \log \left(x^{T}(t) F x(t)\right) \\
\leq \frac{1}{2} \sum_{i=1}^{N} \pi_{i}\left(\gamma_{i}-2 \delta_{i}+\log \frac{\lambda_{\max }(F) \sigma_{i}^{2}}{\lambda_{\min }(F)}\right)<0 .
\end{aligned}
$$

The proof is complete.

\section{Example}

Let $B(t)$ be a one-dimensional Brownian motion. The character measure $v$ of Poisson jump satisfies $v(\mathrm{~d} y)=\zeta \phi(\mathrm{d} y)$, where $\zeta=1.5$ is the intensity of Poisson distribution and $\phi$ is the probability intensity of the standard normal distributed variable $y, F=I_{2}, r(t) \in \mathbb{S}=\{1,2\}$, and

$$
\Gamma=\left(\gamma_{i j}\right)_{2 \times 2}=\left(\begin{array}{cc}
-5 & 5 \\
3 & -3
\end{array}\right) \text {. }
$$

Consider the following nonlinear stochastic system with Markovian switching and Lévy noises:

$$
\begin{array}{r}
\mathrm{d} x(t)=f(x(t), t, r(t)) \mathrm{d} t+g(x(t), t, r(t)) \mathrm{d} B(t) \\
+\int_{Y} H(x(t-), t, r(t-), y) N(\mathrm{~d} t, \mathrm{~d} y),
\end{array}
$$

where

$$
\begin{aligned}
f(x(t), t, 1) & =-2 x(t)+\frac{1}{3} \sin (x(t)), \\
g(x(t), t, 1) & =\frac{1}{2} x(t), \\
f(x(t), t, 2) & =-\frac{7}{2} x(t)+\frac{1}{5} \sin (x(t)), \\
g(x(t), t, 2) & =\frac{1}{4} x(t) \\
H(x(t), t, 1, y) & =\frac{x(t)}{2} \\
H(x(t), t, 2, y) & =-x(t) .
\end{aligned}
$$

Then, we obtain

$$
\begin{aligned}
& \pi_{1}=0.35, \\
& \pi_{2}=0.65, \\
& \gamma_{1}=-9.26, \\
& \gamma_{2}=-5.43, \\
& \delta_{1}=0.25, \\
& \delta_{2}=0.15, \\
& \xi_{1}=1.46, \\
& \xi_{2}=2.10, \\
& \sigma_{1}=2, \\
& \sigma_{2}=3 .
\end{aligned}
$$




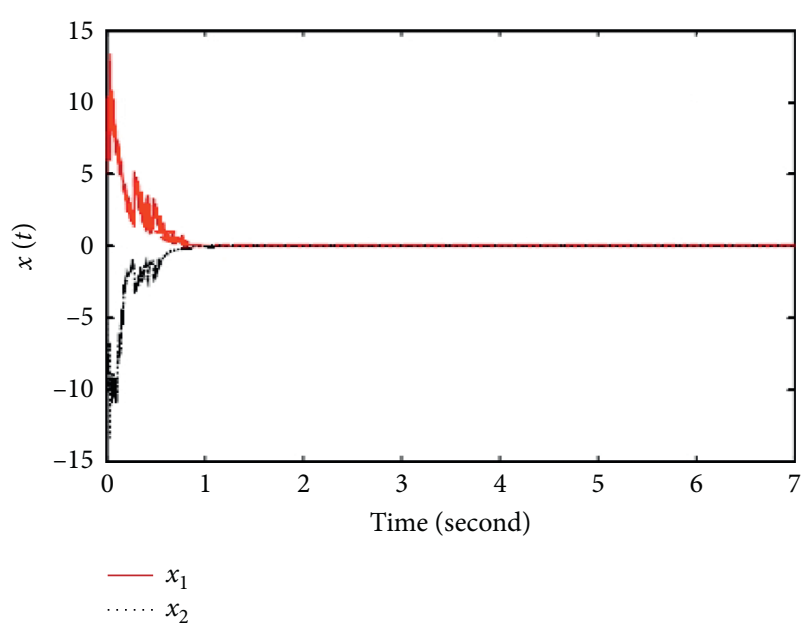

FiguRE 1: State trajectory.

$$
\frac{1}{2} \sum_{i=1}^{2} \pi_{i}\left(\gamma_{i}-2 \delta_{i}+\log \frac{\lambda_{\max }(F) \sigma_{i}^{2}}{\lambda_{\min }(F)}\right)=-0.1565<0 .
$$

Therefore, the system is almost sure exponential stability. Figure 1 shows the simulation results.

\section{Conclusion}

The aim of this paper is to study the existence, uniqueness, and almost sure exponential stability of solution to nonlinear stochastic system with Markovian switching and Lévy noises. The existence of the global unique solution to the system has been derived. Almost sure exponential stability of the solution has been analyzed by the generalized Itô formula, the law of large numbers for local martingale, and the ergodic of Markovian chain. Further research topics will include the stability for the nonlinear delayed stochastic system with Markovian switching and $\alpha$ stable noises [22].

\section{Data Availability}

No data were used to support this study.

\section{Conflicts of Interest}

The authors declare that there are no conflicts of interest.

\section{Acknowledgments}

This work was supported in part by the National Natural Science Foundation of China (Grant no. 61403248).

\section{References}

[1] H. Gao and T. Chen, "Stabilization of nonlinear systems under variable sampling: a fuzzy control approach," IEEE Transactions on Fuzzy Systems, vol. 15, no. 5, pp. 972-983, 2007.

[2] C. Hua, P. Liu, and X. Guan, "Backstepping control for nonlinear systems with time delays and applications to chemical reactor systems," IEEE Transactions on Industrial Electronics, vol. 56, no. 9, pp. 3723-3732, 2009.
[3] J. Bertoin, Lévy Processes, Cambridge University Press, Cambridge, UK, 1996.

[4] D. Applebaum, Lévy Processes and Stochastic Calculus, Cambridge University Press, Cambridge, UK, 2004.

[5] D. Applebaum, Lévy Processes and Stochastic Calculus, Cambridge University Press, Cambridge, UK, 2nd edition, 2008.

[6] H. Y. Wong and P. Guan, "An FFT-network for Lévy option pricing," Journal of Banking \& Finance, vol. 35, no. 4, pp. 988-999, 2011.

[7] J. Liao, H. Shu, and C. Wei, "Pricing power options with a generalized jump diffusion," Communications in StatisticsTheory and Methods, vol. 46, no. 22, pp. 11026-11046, 2017.

[8] G. Wei, Z. Wang, and H. Shu, "Nonlinear $H_{\infty}$ control of stochastic time-delay systems with Markovian switching," Chaos, Solitons \& Fractals, vol. 35, no. 3, pp. 442-451, 2008.

[9] F. Xi, "On the stability of jump-diffusions with Markovian switching," Journal of Mathematical Analysis and Applications, vol. 341, no. 1, pp. 588-600, 2008.

[10] L. Huang and X. Mao, "On almost sure stability of hybrid stochastic systems with mode-dependent interval delays," IEEE Transactions on Automatic Control, vol. 55, no. 8, pp. 1946-1952, 2010.

[11] W. Zhou, D. Tong, Y. Gao et al., "Slide mode and delaydependent adaptive exponential synchronization in $p$ th moment for stochastic delayed neural networks with Markovian switching," IEEE Transactions on Neural Networks and Learning Systems, vol. 23, no. 4, pp. 662-668, 2012.

[12] Y. Wu, S. Yan, M. Fan, and W. Li, "Stabilization of stochastic coupled systems with Markovian switching via feedback control based on discrete-time state observations," International Journal of Robust and Nonlinear Control, vol. 28, no. 1, pp. 247-265, 2018.

[13] M. Li and F. Deng, "Moment exponential input-to-state stability of non-linear switched stochastic systems with Lévy noise," IET Control Theory \& Applications, vol. 12, no. 9, pp. 1208-1215, 2018.

[14] M. Li, L. Liu, and F. Deng, "Input-to-state stability of switched stochastic delayed systems with Lévy noise," Journal of the Franklin Institute, vol. 355, no. 1, pp. 314-331, 2018.

[15] Y. Ren, W. Yin, and R. Sakthivel, "Stabilization of stochastic differential equations driven by G-Brownian motion with feedback control based on discrete-time state observation," Automatica, vol. 95, no. 2, pp. 146-151, 2018.

[16] G. Yin and C. Zhou, "Hybrid switching diffusions properties and applications," Stochastic Modelling and Applied Probability, Springer, Berlin, Germany, 2010.

[17] M. Liu, I. Dassios, and F. Milano, "On the stability analysis of systems of neutral delay differential equations," Circuits, Systems, and Signal Processing, vol. 38, no. 4, pp. 1639-1653, 2019.

[18] X. Wu, Y. Tang, J. Cao et al., "Stability analysis for continuoustime switched systems with stochastic switching signals," IEEE Transactions on Automatic Control, vol. 63, no. 9, pp. 3083-3090, 2017.

[19] X. Li, H. Gao, and K. Gu, "Delay-independent stability analysis of linear time-delay systems based on frequency discretization," Automatica, vol. 70, pp. 288-294, 2016.

[20] Y. Song and W. Xu, "Stability of a gene transcriptional regulatory system under non-Gaussian noise," Chaos, Solitons \& Fractals, vol. 130, p. 109430, 2020.

[21] X. Mao and C. Yuan, Stochastic Differential Equations with Markovian Switching, Imperial College Press, London, UK, 2006.

[22] F. Xi and G. Yin, "Almost sure stability and instability for switching-jump-diffusion systems with state-dependent switching," Journal of Mathematical Analysis and Applications, vol. 400, no. 2, pp. 460-474, 2013. 